\title{
SOUNDING THE ORIGIN OF L-BAND SAR STRIPES IN THE EQUATORIAL IONOSPHERE: COORDINATED OBSERVATION OF ALOS-2 AND AIR GLOW IMAGER
}

\author{
Hiroatsu Sato ${ }^{1}$, Jun Su Kim ${ }^{2}$, Cristiano Max Wrasse ${ }^{3}$, Jonas Rodrigues de Souza ${ }^{3}$ \\ ${ }^{1}$ German Aerospace Center (DLR), Institute of Communications and Navigation, Germany \\ ${ }^{2}$ German Aerospace Center (DLR), Microwaves and Radar Institute, Germany \\ ${ }^{3}$ National Institute for Space Research (INPE), Aeronomy Division, Brazil
}

\begin{abstract}
We study small scale ionospheric effects on L-band SAR images in the equatorial region, known as equatorial stripes or streaks by using ALOS2/PALSAR-2 and ground air glow imager. Our experiment shows the existence of density depletion regions in the ionosphere within the radar geometry when SAR stripe phenomena occurred. This study experimentally demonstrates that the equatorial Lband SAR stripes are associated with plasma irregularities in density depletion regions, although further work is necessary to clarify the formation mechanism of irregularities.
\end{abstract}

Index Terms $-S A R$, ionosphere, scintillation, air glow

\section{INTRODUCTION}

Ionosphere is a major source of propagation error for satellite radio signals. Small scale ionospheric density structures cause rapid changes in satellite radio waves, known as scintillation. Ionospheric scintillation effects on SAR images are typically characterized as image distortion with stripe patterns of signal amplitude. These patterns shows high spatial frequency approximately aligned to the projection of the geomagnetic field onto the SAR image plane. The SAR stripe phenomena was first reported through the Phased Array L-band Synthetic Aperture Radar (PALSAR) instrument on board Advanced Land Observation Satellite (ALOS) observation over the Amazon terrain [1].

Recent observational and simulation studies have improved our knowledge about the origin of the equatorial SAR stripe patterns. Statistical analysis of
ALOS-2 image over south America shows that these ionosphere-induced stripe distortions occur frequently at night time and the pattern can stretch over more than $1000 \mathrm{~km}$ in the low latitude [2]. New observational technique has been developed to estimate the height and the drift velocity of the scintillations from the stripe patterns [3]. Although the occurrence of stripes seem to related to the equatorial plasma bubbles, phase screen modeled simulation results suggests that the stripe patterns maybe related to bottom side sinusoidal irregularity rather than plasma bubbles [4] .

While these recent papers demonstrated that postsunset ionospheric scintillation can cause the stripe structures on L-band SAR image, there has been few direct observation of ionospheric density structures simultaneously by other instruments.

In this paper, we present a coordinated observation campaign of SAR equatorial stripes in north Brazil with ALOS-2 and ground based optical instrument. We use an all sky imager located within the SAR ground image frame to monitor $630-\mathrm{nm}$ air glow. The air glow imager can provide two dimensional image of plasma bubble which appears as depletion of air glow. With this setup we intend to clarify the existence of plasma bubble and its relation to SAR stripes.

\section{EXPERIMENTAL SETUP}

The SAR images used in this study were acquired from ALOS-2/PALSAR-2 operated by the Japan Aerospace Exploration Agency (JAXA). ALOS-2 orbits at approximately $628 \mathrm{~km}$ in altitude at an inclination angle of $97.9^{\circ}$ in a sun-synchronous orbit. The PALSAR-2 instrument operates at different center frequencies around $1257.5 \mathrm{MHz}$. 
The all sky imager used in this paper is installed at Boa Vista $\left(2.49^{\circ} \mathrm{N}, 60.40^{\circ} \mathrm{W}\right)$, north Brazil. The all sky imager is equipped with $180^{\circ}$ wide-angle fish-eye lens, a telecentric lens system and narrow band optical filters to obtain a monochromatic image on a CCD camera [5]. By monitoring the airglow of OI $630 \mathrm{~nm}$, it is possible to acquire two dimensional image of $\mathrm{F}$ region plasma irregularity layer. The acquired image can be transformed into latitude/longitude coordinate with the algorithm based in [6]. The transformed image has $512 \times 512$ pixels and each pixel size corresponds to $2 \mathrm{~km}$. Therefore one all sky image covers $1024 \times 1024 \mathrm{~km}$ in horizontal plane, which allow a good spatial coverage of upper atmosphere during SAR image acquisition time.

\section{OBSERVATION}

Figure 1 illustrates the SAR imaging geometry near Boa Vista on December 29, 2014. Each SAR frame is shown by the red squares. The image center time at the thick red square is 04:16:43 UT (ALOS-2 scene ID = ALOS2032390040-141229, hereafter scene 1). This SAR image involves the location of Boa Vista where the all sky imager is installed. The adjacent scenes on south and north were acquired at 04:16:35 UT and 04:16:51 UT, respectively. Table 1 summarizes the SAR images used this study.

Figure 2 shows the five consecutive SAR images around the scene 1 represented in the subband power images normalized by full band [3]. The subband here is $1 / 16$ th of the total bandwidth. The top side of Figure 2 corresponds to the north of Boa Vista. There are two regions of clear stripes in Figure 2, north and south of Boa vista. The stripe strength is observed to be stronger in the south. Between these stripe regions is very weak or no-stripe region which separates the stripe regions by approximately 40 to $60 \mathrm{~km}$ on the ground image geometry.

Four all sky images of $630 \mathrm{~nm}$ air glow near ALOS2 acquisition times are shown in Figure 3. Each image is recorded approximately 2 minutes apart. The dark band-like structures extending in north-south direction are the signature of depleted electron density region at approximately $250 \mathrm{~km}$ altitude. The band-like structure remains in F layer before and after the SAR acquisition time.

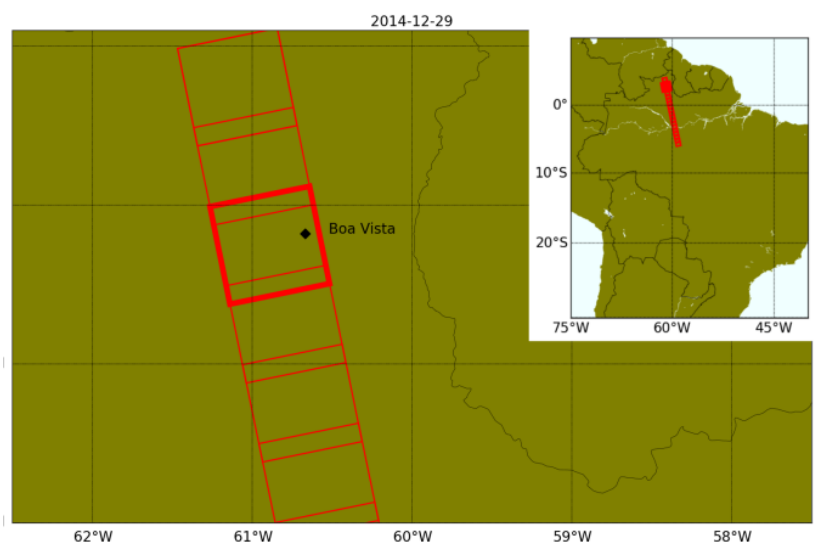

Figure 1. ALOS-2 observation geometry over Boa Vista, Brazil on December 29, 2014. The thick red rectangle corresponds to the ALOS scene ID ALOS2032390040.

\begin{tabular}{|l|l|l|l|}
\hline \multirow{2}{*}{ Scene ID } & \multicolumn{3}{|c|}{ Scene Centre } \\
\cline { 2 - 4 } & UT & Lat. $\left(^{\circ}\right)$ & Lon. $\left(^{\circ}\right)$ \\
\hline ALOS2032390020 & $4: 16: 27$ & 1.753 & -60.68 \\
\hline ALOS2032390030 & $4: 16: 35$ & 2.251 & -60.78 \\
\hline ALOS2032390040 & $4: 16: 43$ & 2.748 & -60.89 \\
\hline ALOS2032390050 & $4: 16: 51$ & 3.246 & -60.99 \\
\hline ALOS2032390060 & $4: 16: 59$ & 3.743 & -61.09 \\
\hline
\end{tabular}

Table 1. ALOS2 scene ID, UT and geographical coordinates of the scene centres of the images used in Figure 1 and Figure 2.

\section{DISCUSSION AND CONCLUSIONS}

The observation result of the ALOS-2 and all sky imager show that both no-stripe region and the dark band structures around $50 \mathrm{~km}$ scale are aligned approximately along the geomagnetic field lines. This implies that plasma irregularities causing SAR stripe may be embedded in the density depletion regions. The dark regions in Figure 3 can be interpreted the signature of (weak) plasma bubble, although other possibilities cannot be excluded since the 4 UT is not typical time for plasma bubble development in this region. 


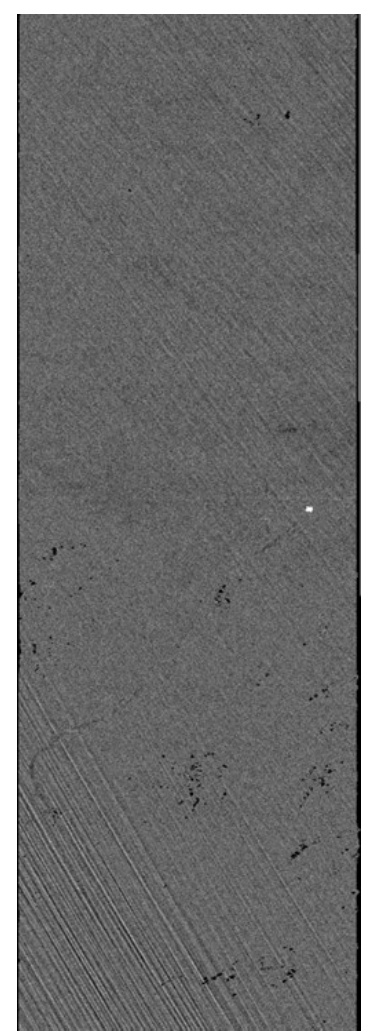

Figure 2. SAR stripes acquisitions over Boa Vista represented in 1/16th subband power images normalized to full-band power. The white dot indicates the location of Boa Vista.

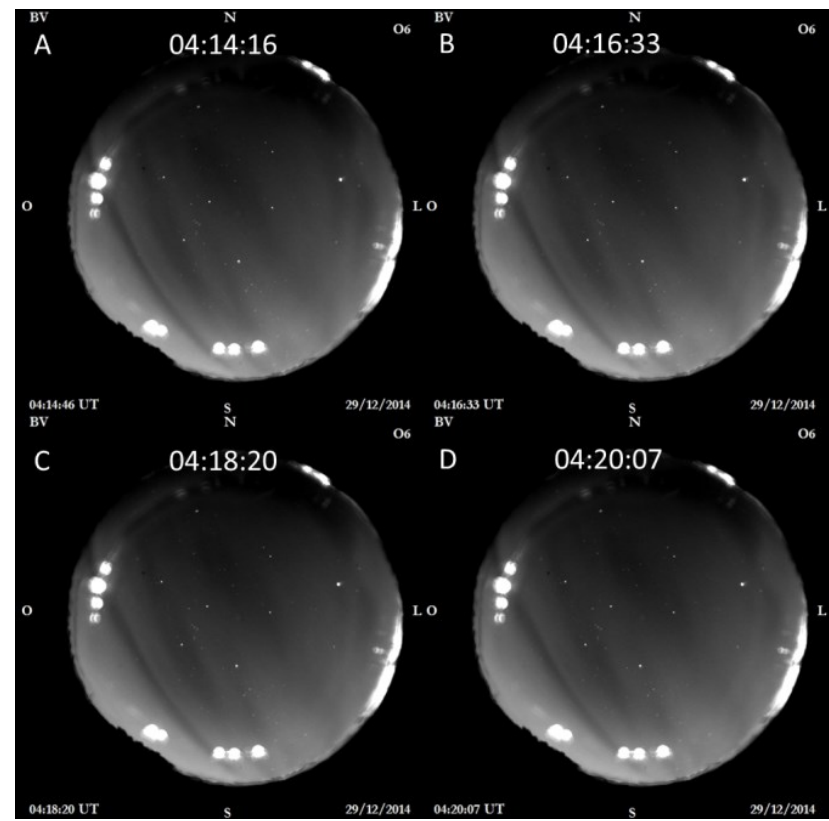

Figure 3. Time series of OI $630 \mathrm{~nm}$ airglow images near ALOS-2 acquisition times.

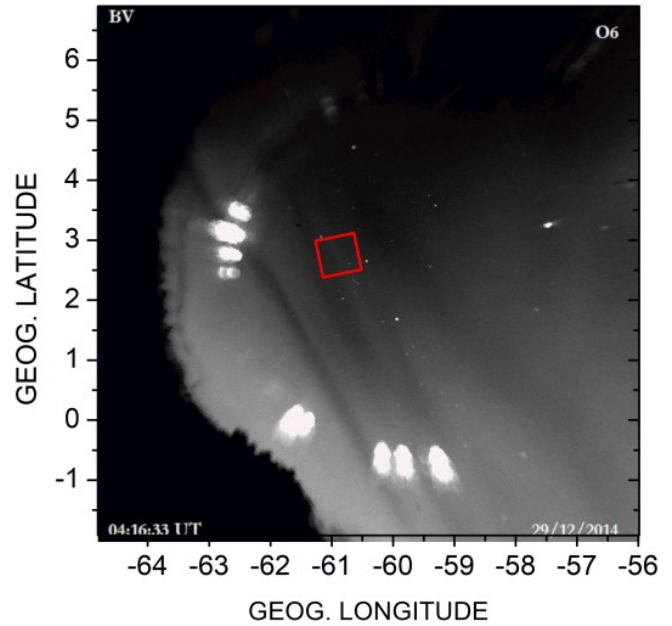

Figure 4. $630 \mathrm{~nm}$ air glow image at 04:16:33 UT mapped on geographical coordinate. The red rectangle corresponds to the ALOS scene ID ALOS2032390040.

In order to compare more closely the location of SAR stripes and the density depletions in the all sky images, the all sky image at 04:16:33 UT is transformed in geographical coordinate in Figure 4. The red square in Figure 4 is the location of SAR ground image of the scene 1 mapped in the all sky image. It is clearly seen that the SAR stripes occurred near the density depletion regions and the spatial scale of stripe separation regions is comparable to the density depletion structure. At the south of the red square, two band-like structures are separated by an air glow band. The width of this white band measured from south-west to north east is approximately $50 \mathrm{~km}$ when mapped on the $250 \mathrm{~km}$ altitude.

A simulation study suggested that the frequency structure of SAR stripes are more close to other type of irregularity such as bottomside sinusoidal irregularities rather than plasma bubble [4]. The existence of such equatorial $F$ region plasma irregularities were first reported by satellite measurements [7]. The scintillation effects of this irregularity were studied for VHF satellite signals. The authors reported that these irregularities are longlived and the scintillation could continue up to 6 hours [8]. These irregularities have not yet been studied extensively in term of the effect on L-band radio 
signals. Our observation shows that plasma density depletions existed under the SAR stripe conditions. Although the sinusoidal irregularities studied by VHF scintillation reported in the literature cannot be verified as the cause of the stripes in this study, some type of bottom side irregularities rather than developed plasma bubble may be the triggering mechanism of L-band SAR stripe. The all sky image in Fgiure 3 and Figure 4 show the density depletions are not well developed into the plasma bubbles.

While the relations between SAR equatorial stripes and density depletions are being addressed by this study, further observational and theoretical studies are necessary to understand the formation mechanism of the irregularities. Complementary observation including GNSS scintillation and TEC measurements will be useful to identify the origin of the SAR stripes.

\section{REFERENCES}

[1] M. Shimada, Y. Muraki, and Y. Otsuka, "Discovery of anoumoulous stripes over the amazon by the PALSAR onboard ALOS satellite," Int. Geosci. Remote Sens. Symp., vol. 2, no. 1, pp. 387-390, 2008.

[2] F. J. Meyer, K. Chotoo, S. D. Chotoo, B. D. Huxtable, and C. S. Carrano, "The Influence of Equatorial Scintillation on L-Band SAR Image Quality and Phase," IEEE Trans. Geosci. Remote Sens., vol. PP, no. 2, pp. 1-12, Feb. 2016.

[3] J. S. Kim, K. P. Papathanassiou, H. Sato, and S. Quegan, "Detection and Estimation of Equatorial Spread F Scintillations Using Synthetic Aperture Radar," IEEE Trans. Geosci. Remote Sens., vol. 55, no. 12, pp. 6713-6725, 2017.

[4] C. S. Carrano, K. M. Groves, and R. G. Caton, "Simulating the impacts of ionospheric scintillation on L band SAR image formation," Radio Sci., vol. 47, no. 4, p. n/a--n/a, 2012.

[5] H. Takahashi et al., "Plasma bubble monitoring by TEC map and $630 \mathrm{~nm}$ airglow image," $J$. Atmos. Solar-Terrestrial Phys., vol. 130-131, pp. 151-158, Aug. 2015.

[6] F. J. Garcia, M. J. Taylor, and M. C. Kelley, "Two-dimensional spectral analysis of mesospheric airglow image data," Appl. Opt., vol. 36, no. 29, p. 7374, 1997.

[7] C. E. Valladares, W. B. Hanson, J. P. McClure, and B. L. Cragin, "Bottomside sinusoidal irregularities in the equatorial F region," $J$. Geophys. Res., vol. 88, no. A10, p. 8025, 1983.

[8] S. Basu, S. Basu, C. E. Valladares, A. DasGupta, and H. E. Whitney, "Scintillations associated with bottomside sinusoidal irregularities in the equatorial $\mathrm{F}$ region," $J$. Geophys. Res. Sp. Phys., vol. 91, no. A1, pp. 270-276, 1986. 